\title{
On the irrationality of generalized $q$-logarithm
}

\author{
Wadim Zudilin*
}

\author{
${ }^{*}$ Correspondence: \\ wzudilin@gmail.com \\ School of Mathematical and \\ Physical Sciences, The University \\ of Newcastle, Callaghan, NSW \\ 2308, Australia
}

To my good friends and fine colleagues Peter Bundschuh and Keijo Väänänen.

$$
\begin{aligned}
& \text { Abstract } \\
& \text { For integer } p,|p|>1 \text {, and generic rational } x \text { and } z \text {, we establish the irrationality of the } \\
& \text { series } \\
& \qquad \ell_{p}(x, z)=x \sum_{n=1}^{\infty} \frac{z^{n}}{p^{n}-x} . \\
& \text { It is a symmetric }\left(\ell_{p}(x, z)=\ell_{p}(z, x)\right) \text { generalization of the } q \text {-logarithmic function }(x=1 \\
& \text { and } p=1 / q \text { where }|q|<1) \text {, which in turn generalizes the } q \text {-harmonic series } \\
& (x=z=1) \text {. Our proof makes use of the Hankel determinants built on the Padé } \\
& \text { approximations to } \ell_{p}(x, z) \text {. }
\end{aligned}
$$

Keywords: Irrationality, Rational approximation, q-Logarithm, q-Harmonic series, Basis hypergeometric series, Padé approximation, Hankel determinant

Mathematics Subject Classification: Primary 11J72; Secondary 11C20, 33D15, 41A20

\section{Historical notes}

In 1827, a problem was published about the series

$$
q+2 q^{2}+2 q^{3}+3 q^{4}+2 q^{5}+4 q^{6}+2 q^{7}+\cdots=\sum_{n=1}^{\infty} \frac{q^{n}}{1-q^{n}}
$$

(see [16, Aufgabe16, p. 99]). It was pointed out that J. Lambert had shown that the coefficient of $q^{n}$ in the series counts the number of divisors of $n$, so that the coefficient 2 may only appear when the exponent of $q$ is a prime number. The question asked was to find a "finite expression" for the $q$-series. The latter converges inside the disc $|q|<1$ and is usually referred by the name of $q$-harmonic series, because multiplication of the right-hand side by $1-q$ and the termwise limits $(1-q) \times q^{n} /\left(1-q^{n}\right) \rightarrow 1 / n$ as $q \rightarrow 1^{-}$ formally assign to it the divergent harmonic series (see [27, Division 2, Problem 32] for a precise limiting behaviour of $(1)$ as $q \rightarrow 1^{-}$). As we are aware these days, the series (1) is quite independent of other known $q$-series, even of its relatives [29], so it is still an open problem to figure out what other functions can be linearly or algebraically linked to it. However, as first observed by Th. Clausen in the final paragraph of [15], one can easily

(c) 2016 The Author(s). This article is distributed under the terms of the Creative Commons Attribution 4.0 International License (http://creativecommons.org/licenses/by/4.0/), which permits unrestricted use, distribution, and reproduction in any medium, provided you give appropriate credit to the original author(s) and the source, provide a link to the Creative Commons license, and indicate if changes were made. 
accelerate the convergence of the $q$-harmonic series:

$$
\sum_{n=1}^{\infty} \frac{q^{n}}{1-q^{n}}=\sum_{n=1}^{\infty} q^{n^{2}} \frac{1+q^{n}}{1-q^{n}}
$$

(see also [28, Division 8, Problem 74]). This computationally practical formula does not hint at an approach to the arithmetic properties, like the irrationality and transcendence, of the values of $q$-harmonic series. Curiously enough, the previous problem (Aufgabe 15) on the list in [16] requests about the irrationality of the numbers $\pi^{n}$ for integer $n \geq 2$.

The first proof of the irrationality of the $q$-harmonic series for $q$ of the form $q=1 / p$, where $p>1$ is an integer, was recorded by P. Erdös [18] in 1948. His argument uses the non-periodicity of base $p$ expansion of the number in question (and so, certain delicate properties of the number-of-divisors function) and cannot be extended to deal with the more general analytical function

$$
L_{q}(z)=\sum_{n=1}^{\infty} \frac{q^{n} z^{n}}{1-q^{n}}, \quad|z|<1, \quad|q|<1,
$$

known as the $q$-logarithm. Independently, J.-P. Bézivin [3] in 1988 and P. Borwein [7] in 1991 pushed forward different analytical methods to establish the irrationality of the series for rational $z$ and $q$ of the form $q=1 / p$ with $p \in \mathbb{Z} \backslash\{0, \pm 1\}$, though Bézivin's paper did not mention $L_{q}(z)$ specifically-it was observed only later [9] that the general results from [3] imply the irrationality. The advantage of Borwein's method $[7,8]$, in which he uses the Padé approximations to (2), is that it allows one to "measure" the irrationality of the numbers in question; this quantitative counterpart is absent in Erdős' method [18] and it was also absent in Bézivin's original method [3,4] until the recent work [30] of I. Rochev (see [31] for a further development). The Padé approximation technique as originated in $[7,8]$ was significantly generalized and extended in later works (for example, $[1,9-13,17,22,24,25,32,34,36,37]$ to list a few) to sharpen the irrationality measures of the values of $q$-harmonic and $q$-logarithm series as well as to prove the irrationality and linear independence results for some close relatives of the series.

Bézivin's method from [3,4] is capable of dealing with a class of generalized $q$ hypergeometric functions that are quite different from (2). It is a "lucky accident" that the $q$-logarithm appears as the quotient of two such functions - it happens to be the logarithmic derivative of the so-called exponential function

$$
E_{q}(z)=\sum_{n=0}^{\infty} \frac{q^{n(n+1) / 2} z^{n}}{\prod_{j=1}^{n}\left(1-q^{j}\right)}=\prod_{n=1}^{\infty}\left(1+q^{n} z\right) ;
$$

namely, $L_{q}(z)=z E_{q}^{\prime}(-z) / E_{q}(-z)$ where the derivative is with respect to $z$. Though the method from $[3,4]$ underwent modifications and generalizations in the later works of Bézivin himself and other authors [5,6,14,23,30,31], it essentially serves the original class of functions.

The generalization of the $q$-logarithm we discuss here is given by

$$
\begin{aligned}
\ell_{p}(x, z) & =x \sum_{n=1}^{\infty} \frac{q^{n} z^{n}}{1-q^{n} x}=\sum_{m, n>0} q^{m n} x^{m} z^{n} \\
& =x \sum_{n=1}^{\infty} \frac{z^{n}}{p^{n}-x}, \quad|x|<|p| \text { and }|z|<|p|,
\end{aligned}
$$

where $q=1 / p$ is inside the unit disc. In spite of the several remarkable properties of the function, including its symmetry $\ell_{p}(x, z)=\ell_{p}(z, x)$ and the functional equation 


$$
\ell_{p}(x, z)=\frac{x z}{p-x}+z \ell_{p}\left(\frac{x}{p}, z\right)
$$

it has not attracted much attention because of its overall arithmetic toughness. The generalized $q$-logarithm (3) was a topic of our joint investigation [13] with P. Bundschuh; there we constructed explicitly Padé-type approximations to the function and applied them to the cases when $x$ and $z$ are $q$-multiplicatively dependent-a quotient of the form $x^{k} / z^{m}$ is an integral power of $q$ for some integers (not simultaneously zero) $k$ and $m$. Some fundamental issues with the Padé approximations to (3) do not allow to apply the latter for generic $x$ and $z$ even under the standard setting $p \in \mathbb{Z} \backslash\{0\}$. Apart from the $q$-logarithm specialization $(x=1)$, some other cases of (3) are of historical importance, like

$$
\pi_{q}=1-4 \ell_{p^{2}}(p,-1)=1+4 \sum_{n=0}^{\infty} \frac{(-1)^{n} q^{2 n+1}}{1-q^{2 n+1}}=1+4 \sum_{n=1}^{\infty} \frac{q^{n}}{1+q^{2 n}}=\left(\sum_{n=-\infty}^{\infty} q^{n^{2}}\right)^{2} .
$$

Several such instances, under the $q$-multiplicative dependence, are studied in $[12,13]$ from arithmetic perspectives, both qualitatively and quantitatively.

As no representation of the function (3) through the Bézivin-type functions is known, the method from $[3,4]$ and its generalizations are not applicable for arithmetic studies of the values of the function. Other available techniques for $q$-hypergeometric functions based on the ideas of the classical Siegel-Shidlovsky method, for example [2], fail to apply to the function $\ell_{p}(x, z)$ for similar reasons.

\section{Principal results}

In this note we combine the Padé approximation construction from [13] with a version of Bézivin's method as developed in [23] to prove the following general result.

Theorem 1 For integer $p$ and nonzero rational $x, z$ such that $|p|>1, x \notin\left\{p, p^{2}, p^{3}, \ldots\right\}$ and $|z|<|p|$ (to ensure the convergence of the series in (3)), the value of $\ell_{p}(x, z)$ is irrational.

A general framework of the approach we use in our proof of the theorem is outlined in [38].

We now introduce the $q$-hypergeometric function

$$
\begin{aligned}
\mathcal{F}_{p}(x, y, z) & =\sum_{n=0}^{\infty} \frac{\prod_{k=1}^{n}\left(p^{k}-1\right)}{\prod_{k=1}^{n}\left(p^{k}-x\right)\left(p^{k}-y\right)} \cdot(-p z)^{n} \\
& =\sum_{n=0}^{\infty} \frac{(-1)^{n} \prod_{k=1}^{n}\left(1-q^{k}\right)}{\prod_{k=1}^{n}\left(1-q^{k} x\right)\left(1-q^{k} y\right)} \cdot q^{n(n-1) / 2} z^{n}, \quad|x|<|p| \text { and }|z|<|p|,
\end{aligned}
$$

where $q=1 / p$. This is an exemplar function to which the known arithmetic methods $[2,3]$ are not applicable because of the presence of the $q$-Pochhammer product $(q ; q)_{n}=$ $\prod_{k=1}^{n}\left(1-q^{k}\right)$ in the numerator of the $n$-th term of the series. A hypergeometric identity and Theorem 1 allow us to establish the following corollary.

Theorem 2 The identity

$$
\mathcal{F}_{p}(x, z, x z)=\frac{(1-x)(1-z)}{p x z} \ell_{p}(p x, p z)
$$

is valid. In particular, for an integer $p \neq 0, \pm 1$ and rational $x$ and $z$ such that $0<|x|$, $|z|<1$, the value of the hypergeometric function $\mathcal{F}_{p}(x, z, x z)$ is irrational. 
In particular, for (1) we obtain

$$
\sum_{n=1}^{\infty} \frac{q^{n}}{1-q^{n}}=\sum_{n=1}^{\infty} \frac{(-1)^{n-1} q^{n(n+1) / 2}}{\left(1-q^{n}\right) \prod_{k=1}^{n}\left(1-q^{k}\right)}
$$

which resembles the counterpart of the $q$-harmonic series in the " $\frac{1}{2}$-logarithmic derivative of the Dedekind eta-function" [33, Remark on p. 952].

Finally, we remark that the results can be given for non-integer $p=r / s,|p|>1$, as well under a customary in such situations assumption $\log |r|>c \log |s|$ for some computable constant $c>0$. Furthermore, irrationality measures for the numbers in question can be produced showing that they are not Liouvillian. To make our exposition clear and concise, we do not pursue such quantitative issues here but place a related comment after our proof of Theorem 1, at the end of Sect. 4.

The standard $q$-notation [20] we use below include the (multiple) $q$-Pochhammer symbol

$$
\begin{aligned}
\left(a_{1}, a_{2}, \ldots, a_{r} ; q\right)_{n}= & \prod_{j=1}^{n}\left(1-a_{1} q^{j-1}\right)\left(1-a_{2} q^{j-1}\right) \ldots\left(1-a_{r} q^{j-1}\right) \\
& \text { for } n=0,1,2, \ldots, \infty
\end{aligned}
$$

(the empty product for $n=0$ is set to be 1 ), the $q$-binomial coefficients

$$
\left[\begin{array}{l}
n \\
k
\end{array}\right]_{q}=\frac{(q ; q)_{n}}{(q ; q)_{k} \cdot(q ; q)_{n-k}} \text { for } k=0,1, \ldots, n \text { and } n=0,1,2, \ldots,
$$

and the basic hypergeometric function

$$
{ }_{r} \phi_{s}\left(\begin{array}{c}
a_{1}, a_{2}, \ldots, a_{r} \\
b_{1}, \ldots, b_{s}
\end{array} \mid q, z\right)=\sum_{n=0}^{\infty} \frac{\left(a_{1}, a_{2}, \ldots, a_{r} ; q\right)_{n}}{\left(q, b_{1}, \ldots, b_{s} ; q\right)_{n}}\left((-1)^{n} q^{n(n-1) / 2}\right)^{s+1-r} z^{n} .
$$

\section{Padé approximations}

In [13], the following Padé-type approximations to the function (3) were constructed:

$$
\begin{aligned}
I_{n}(q, x, z) & =(x z)^{n+1} \sum_{t=0}^{\infty} \frac{\left(q^{t+1} ; q\right)_{n} \cdot q^{(n+1) t} z^{t}}{\left(q^{n+1+t} x ; q\right)_{n+1}} \\
& =(x z)^{n+1} \frac{(q ; q)_{n}}{\left(q^{n+1} x ; q\right)_{n+1}} \cdot{ }_{2} \phi_{1}\left(\begin{array}{c}
q^{n+1}, q^{n+1} x \\
q^{2 n+2} x
\end{array} \mid q, q^{n+1} z\right)
\end{aligned}
$$

(we choose $m=n$ in the notation of that paper). It is shown in [13, Lemma 2] that

$$
I_{n}(q, x, z)=A_{n}(p) \ell_{p}(x, z)-B_{n}(p)-C_{n}(p),
$$

where $A_{n}(p)=A_{n}(p, x), B_{n}(p)=B_{n}(p, x)$ and $C_{n}(p)=C_{n}(p, x, z)$ are given by the formulae

$$
\begin{aligned}
& A_{n}(p)=p^{(n+1)(3 n+2) / 2} x^{-n} \sum_{k=0}^{n}(-1)^{k} p^{k(k+1) / 2} \frac{\prod_{j=1}^{n}\left(p^{k+j}-x\right)}{(p ; p)_{k}(p ; p)_{n-k}} z^{-k} \\
& B_{n}(p)=p^{(n+1)(3 n+2) / 2} x^{-n+1} \sum_{k=0}^{n}(-1)^{k} p^{k(k+1) / 2} \frac{\prod_{j=1}^{n}\left(p^{k+j}-x\right)}{(p ; p)_{k}(p ; p)_{n-k}} \sum_{l=1}^{k} \frac{z^{l-k}}{p^{l}-x}, \\
& C_{n}(p)=p^{(n+1)(3 n+2) / 2} z \sum_{l=0}^{n-1} \frac{x^{-l}}{p^{n-l}-z} \sum_{k=0}^{l}(-1)^{k}\left[\begin{array}{c}
n \\
k
\end{array}\right]_{p}\left[\begin{array}{c}
n+l-k \\
n
\end{array}\right]_{p} p^{(n-k)(n-k+1) / 2} x^{k} .
\end{aligned}
$$


Denote

$$
D_{n}(p)=D_{n}(p, x, z)=(x z)^{n} \frac{\prod_{j=1}^{n}\left(p^{j}-1\right)\left(p^{j}-x\right)\left(p^{j}-z\right)}{p^{3 n(n+1) / 2}}=(x z)^{n}(q, q x, q z ; q)_{n} .
$$

It follows that

$$
\begin{gathered}
\widetilde{A}_{n}(p)=D_{n}(p) A_{n}(p) \in \mathbb{Z}[p, x, z], \quad \widetilde{B}_{n}(p)=D_{n}(p) B_{n}(p) \in \mathbb{Z}[p, x, z] \\
\text { and } \widetilde{C}_{n}(p)=D_{n}(p) C_{n}(p) \in \mathbb{Z}[p, x, z],
\end{gathered}
$$

with the degrees of the polynomials in $x$ and $z$ not exceeding $2 n$. We now define

$$
v_{n}(\mu)=v_{n}(\mu ; p, x, z)=\widetilde{A}_{n}(p) \mu-\widetilde{B}_{n}(p)-\widetilde{C}_{n}(p),
$$

a linear form in $\mu$ with coefficients from $\mathbb{Z}[p, x, z]$, and summarise the above as follows:

$$
v_{n}\left(\ell_{p}(x, z)\right)=(x z)^{2 n+1} \frac{(q, q, q x, q z ; q)_{n}}{\left(q^{n+1} x ; q\right)_{n+1}} \cdot{ }_{2} \phi_{1}\left(\begin{array}{c}
q^{n+1}, q^{n+1} x \\
q^{2 n+2} x
\end{array} \mid q, q^{n+1} z\right) .
$$

Though the symmetry of this expression is not transparent, it follows from one of Heine's classical transformations, namely, from [20, Eq. (III.1)].

\section{Hankel determinants}

For $n=1,2, \ldots$, define the Hankel determinants

$$
V_{n}(\mu)=V_{n}(\mu ; p, x, z)=\operatorname{det}_{0 \leq j, l \leq n-1}\left(v_{j+l}(\mu)\right) .
$$

These are polynomials in $\mathbb{Z}[\mu, p, x, z]$ of degree $n$ in $\mu$ and of degree at most $2 n(n-1)$ in each of the variables $x$ and $z$. Choosing

$$
v_{n}^{*}=(x z)^{-2 n-1} v_{n}\left(\ell_{p}(x, z)\right)=\frac{(q, q, q x, q z ; q)_{n}}{\left(q^{n+1} x ; q\right)_{n+1}} \cdot{ }_{2} \phi_{1}\left(\begin{array}{c}
q^{n+1}, q^{n+1} x \\
q^{2 n+2} x
\end{array} \mid q, q^{n+1} z\right),
$$

we obtain

$$
V_{n}\left(\ell_{p}(x, z)\right)=\operatorname{det}_{0 \leq j, l \leq n-1}\left((x z)^{2 j+2 l+1} v_{j+l}^{*}\right)=(x z)^{n(2 n-1)} \operatorname{det}_{0 \leq j, l \leq n-1}\left(v_{j+l}^{*}\right)=(x z)^{n(2 n-1)} V_{n}^{*} .
$$

It is immediate from (6) that the $q$-expansion of $v_{n}^{*}$ starts from the constant term, $v_{n}^{*}=$ $1+O(q)$, so that $v_{n}^{*}=|p|^{O(n)}$ as $n \rightarrow \infty$ (see also [13, Lemma 5]). Our crucial observation here is that the $q$-expansion of $V_{n}^{*}$ starts from at least $q^{n(n-1)(2 n-1) / 6}$, so that $\left|V_{n}^{*}\right| \leq$ $|q|^{n^{3} / 3} \exp \left(O\left(n^{2}\right)\right)$. In order to see that we introduce the backward shift operator $N: v_{n} \mapsto$ $v_{n-1}$ on the indices, its zeroth power $I: v_{n} \mapsto v_{n}$ - the identity operator, and define

$$
D_{l}=(N ; q)_{l}=\prod_{j=0}^{l-1}\left(I-q^{j} N\right)=\sum_{k=0}^{l}(-1)^{k} q^{k(k-1) / 2}\left[\begin{array}{l}
l \\
k
\end{array}\right]_{q} N^{k}
$$

Lemma 1 Let $H(q)=1+\sum_{r=1}^{\infty} a_{r} q^{r}$ be a formal power series starting from 1 and $t a$ nonnegative integer. Define

$$
w_{n}=w_{n}(H, t)=q^{(n+1) t} \prod_{k=1}^{n} H\left(q^{k}\right) .
$$

Then for $n \geq l$ the q-expansion of $D_{l} w_{n}$ starts at least from $n l-l(l-1) / 2$. 
Proof We extend the action of our operators to the linear spaces

$$
\mathcal{W}_{n}=\bigoplus_{m=0}^{\infty}\left\{P_{m}(q) w_{n}(H, m): P_{m}(q) \in \mathbb{C}[q]\right\} \subset \mathbb{C}[[q]]
$$

with the goal to demonstrate, by induction on $l$, that the image of an element from $\mathcal{W}_{n}$ under $D_{l}$ belongs to $q^{n l-l(l-1) / 2} \mathcal{W}_{n-l}$ for $l \neq n$. In particular, this will imply the lemma.

The induction base $(l=0)$ is trivial, because $n l-l(l-1) / 2=0$ for any $n$ in this case. Assume that the required statement is established for an $l$, so that for each integer $t \geq 0$ we have

$$
D_{l} w_{n}(H, t)=q^{n l-l(l-1) / 2} \sum_{m=0}^{\infty} P_{m}(q) w_{n-l}(H, m)
$$

with some $P_{m}(q)=P_{m, n, l, t}(q) \in \mathbb{C}[q]$. Since $D_{l+1}=\left(I-q^{l} N\right) D_{l}$, in order to verify the statement for $l+1 \leq n$ we need to demonstrate that $\left(I-q^{l} N\right)\left(q^{n l-l(l-1) / 2} w_{n-l}(H, m)\right)$ belongs to $q^{n(l+1)-l(l+1) / 2} \mathcal{W}_{n-l-1}$ for any $m \geq 0$. We have

$$
\begin{aligned}
(I- & \left.q^{l} N\right)\left(q^{n l-l(l-1) / 2} w_{n-l}(H, m)\right) \\
& =q^{n l-l(l-1) / 2} \cdot q^{(n-l+1) m} \prod_{k=1}^{n-l} H\left(q^{k}\right)-q^{l} \cdot q^{(n-1) l-l(l-1) / 2} \cdot q^{(n-l) m} \prod_{k=1}^{n-l-1} H\left(q^{k}\right) \\
& =q^{n(l+1)-l(l+1) / 2} \cdot\left(q^{m} H\left(q^{n-l}\right)-1\right) \cdot q^{(n-l)(m-1)} \prod_{k=1}^{n-l-1} H\left(q^{k}\right) \\
& =q^{n(l+1)-l(l+1) / 2}\left(\left(-1+q^{m}\right) w_{n-l-1}(H, m-1)+q^{m} \sum_{r=1}^{\infty} a_{r} w_{n-l-1}(H, m+r-1)\right),
\end{aligned}
$$

with the term $\left(-1+q^{m}\right) w_{n-l-1}(H, m-1)$ absent when $m=0$. This completes the proof of the inductive step, hence of the whole statement and the lemma.

We remark that Lemma 1 applies to general hypergeometric terms

$$
\frac{\left(a_{1}, \ldots, a_{r} ; q\right)_{n}}{\left(b_{1}, \ldots, b_{s} ; q\right)_{n}} q^{(n+1) t}, \quad \text { where } t \text { is a nonnegative integer, }
$$

hence to the (finite or infinite) sums of such terms over $t$, as they belong to the vector space $\mathcal{W}_{n}$. In particular, it applies to

$$
v_{n}^{*}=\sum_{t=0}^{\infty} z^{t} \frac{\left(q, q x, q z, q^{t+1} ; q\right)_{n} \cdot q^{(n+1) t}}{\left(q^{n+1+t} x ; q\right)_{n+1}},
$$

so that the $q$-expansion of $D_{l} v_{n}^{*}$ starts at least from $q^{n l-l(l-1) / 2}$ for any $l \leq n$. Acting on the $j$-th row of the matrix $\left(v_{j+l}^{*}\right)_{0 \leq j, l \leq n-1}$ by $D_{j}$, which results in the elementary manipulations of the rows in accordance with the definition (7) of $D_{j}$, we get the newer matrix $\left(a_{j, l}\right)_{0 \leq j, l \leq n-1}$ of the same determinant $V_{n}^{*}$, whose entries in the $j$-th row have $q$-order at least $n j-j(j-1) / 2$. Thus,

$$
\operatorname{ord}_{q} V_{n}^{*} \geq \sum_{j=0}^{n-1}\left((n-1) j-\frac{j(j-1)}{2}\right)=\frac{n(n-1)(2 n-1)}{6} .
$$

The details of the derivation of the estimate

$$
\left|V_{n}^{*}\right| \leq|q|^{n^{3} / 3} \exp \left(O\left(n^{2}\right)\right) \quad \text { as } n \rightarrow \infty
$$

from $\operatorname{ord}_{q} V_{n}^{*} \geq n^{3} / 3+O\left(n^{2}\right)$ can be borrowed from the proofs of Lemma 2 and Proposition 3 in [23]. Therefore,

$$
\left|V_{n}\left(\ell_{p}(x, z)\right)\right| \leq|p|^{-n^{3} / 3} \exp \left(O\left(n^{2}\right)\right) \quad \text { as } \quad n \rightarrow \infty .
$$


Proof of Theorem 1 If $\ell_{p}(x, z)$ were rational then $\left(x_{0} z_{0}\right)^{2 n(n-1)} V_{n}\left(\ell_{p}(x, z)\right)$ would be an integer for all $n \geq 0$, where $x_{0}$ and $z_{0}$ denote the denominators of $x$ and $z$. It follows from Kronecker's criterion [28, Division 7,Problem 24] and the fact that the series

$$
\sum_{n=0}^{\infty} v_{n}\left(\ell_{p}(x, z)\right) y^{n}=x z \sum_{t=0}^{\infty}(q z)^{t} \sum_{n=0}^{\infty} \frac{\left(q, q x, q z, q^{t+1} ; q\right)_{n} \cdot\left(q^{t} x^{2} z^{2} y\right)^{n}}{\left(q^{n+1+t} x ; q\right)_{n+1}}
$$

is not rational (as the interior basic hypergeometric series are not), that this integer is different from zero for infinitely many $n$, so that

$$
\left|\left(x_{0} z_{0}\right)^{2 n(n-1)} V_{n}\left(\ell_{p}(x, z)\right)\right| \geq 1
$$

for such $n$. The resulting estimate contradicts the bound in (8) for sufficiently large $n$, hence $\ell_{p}(x, z)$ must be irrational.

Another proof of Theorem 1 can be given, without the reference to the Hankel determinants and Kronecker's criterion. It follows from the definition (7) and Lemma 1 that

$$
\begin{aligned}
v_{n}^{\prime}(\mu) & =(x z)^{2 n+1} p^{n(n-1) / 2} \sum_{k=0}^{n}(-1)^{k} p^{-k(k-1) / 2-k(n-k)}\left[\begin{array}{l}
n \\
k
\end{array}\right]_{p}(x z)^{k-2 n-1} v_{2 n-k}(\mu) \\
& =\sum_{k=0}^{n}(-1)^{k} p^{(n-k)(n-k-1) / 2}\left[\begin{array}{l}
n \\
k
\end{array}\right]_{p}(x z)^{k} v_{2 n-k}(\mu) \in \mathbb{Z}[p, x, z] \mu+\mathbb{Z}[p, x, z]
\end{aligned}
$$

for all $n$ and $v_{n}^{\prime}\left(\ell_{p}(x, z)\right)=(x z)^{2 n+1} p^{n(n-1) / 2} D_{n} v_{2 n}^{*}$ satisfies $\operatorname{ord}_{q} v_{n}^{\prime} \geq n(n+1)$, hence is bounded:

$$
\left|v_{n}^{\prime}\left(\ell_{p}(x, z)\right)\right| \leq|p|^{-n^{2}} \exp \left(o\left(n^{2}\right)\right) .
$$

Assuming the nonvanishing $v_{n}^{\prime}\left(\ell_{p}(x, z)\right) \neq 0$ is proven for a sufficiently dense sequence of indices $n$, the argument allows not only to demonstrate the irrationality of $\ell_{p}(x, z)$ but also to show that it is not a Liouville number by giving the related estimate for its irrationality exponent.

\section{A hypergeometric identity}

Before going into details of the proof of identity (5) in Theorem 2, we would like to mention that the inspiration for it comes from the paper [24] by T. Matala-aho. A key identity there is

$$
(1-t) \sum_{n=0}^{\infty} \frac{t^{n}}{(q b ; q)_{n}}=\sum_{n=0}^{\infty} \frac{q^{n^{2}}(b t)^{n}}{(q b, q t ; q)_{n}}, \quad|q|<1, \quad|t|<1 ;
$$

he then studies in [24] arithmetic properties of the series on the right-hand side of (9) by applying Padé-type techniques to the left-hand side. Identity (9) is classical and established in [19, Eq. (12.3)]. A natural generalization of (9), namely the form (11) from the proof below, was kindly communicated to us by C. Krattenthaler [21].

Proof of Theorem 2 Let us start with [20, Eq. (III.9)],

$$
{ }_{3} \phi_{2}\left(\begin{array}{c}
a, b, c \\
d, e
\end{array} \mid q, \frac{d e}{a b c}\right)=\frac{(e / a, d e /(b c) ; q)_{\infty}}{(e, d e /(a b c) ; q)_{\infty}} \cdot{ }_{3} \phi_{2}\left(\begin{array}{c}
a, d / b, d / c \\
d, d e /(b c)
\end{array} \mid q, \frac{e}{a}\right) .
$$

Letting $b$ tend to $\infty$ we obtain

$$
{ }_{2} \phi_{2}\left(\begin{array}{c}
a, c \\
d, e
\end{array} \mid q, \frac{d e}{a c}\right)=\frac{(e / a ; q)_{\infty}}{(e ; q)_{\infty}} \cdot{ }_{2} \phi_{1}\left(\begin{array}{c}
a, d / c \\
d
\end{array} \mid q, \frac{e}{a}\right) .
$$


Further, letting $c$ tend to $\infty$ we deduce

$$
{ }_{1} \phi_{2}\left(\begin{array}{c|c}
a \\
d, e
\end{array} \mid q, \frac{d e}{a}\right)=\frac{(e / a ; q)_{\infty}}{(e ; q)_{\infty}} \cdot{ }_{2} \phi_{1}\left(\begin{array}{c|c}
a, 0 & q, \frac{e}{a} \\
d & .
\end{array}\right.
$$

The particular case $a=q, d=b q$ and $e=t q$ of (11) is exactly identity (9).

Setting $x=d / c, z=e / a$ in the intermediate equation (10) we may write it as follows:

$$
\frac{(z ; q)_{\infty}}{(a z ; q)_{\infty}} \cdot{ }_{2} \phi_{1}\left(\begin{array}{c}
a, x \\
c x
\end{array} \mid q, z\right)={ }_{2} \phi_{2}\left(\begin{array}{c}
a, c \\
c x, a z
\end{array} \mid q, x z\right) .
$$

Then taking $a=c=q$ we obtain

$$
\begin{aligned}
(1-x) \sum_{n=0}^{\infty} \frac{z^{n}}{1-q^{n} x} & ={ }_{2} \phi_{1}\left(\begin{array}{c}
q, x \\
q x
\end{array} \mid q, z\right)=\frac{(q z ; q)_{\infty}}{(z ; q)_{\infty}} \cdot{ }_{2} \phi_{2}\left(\begin{array}{c}
q, q \\
q x, q z
\end{array} \mid q, x z\right) \\
& =\frac{1}{1-z} \sum_{n=0}^{\infty} \frac{(q ; q)_{n}}{(q x, q z ; q)_{n}} q^{n(n-1) / 2}(-x z)^{n} .
\end{aligned}
$$

Finally, performing simple manipulations we cast the resulting identity as (5). This concludes our proof of the theorem.

\section{Related questions}

As already pointed out, there are no general methods at present to prove the irrationality of the values of $q$-hypergeometric series like (4), when $q$-Pochhammer products appear in the numerators of the terms, for a sufficiently generic set of parameters.

A different from (4) three-parameter generalization of $\ell_{p}(x, z)$ can be considered, which is suggested by rational Padé-type approximations in $[10,11,32,34,37]$ that generalize the approximations to the $q$-logarithm function. Namely, we introduce the function

$$
\mathcal{L}_{p}(x, y, z)=\sum_{n=1}^{\infty} \frac{p^{n} z^{n}}{\left(p^{n}-x\right)\left(p^{n}-y\right)}= \begin{cases}\frac{\ell_{p}(x, z)-\ell_{p}(y, z)}{x-y} & \text { if } x \neq y \\ \frac{\mathrm{d}}{\mathrm{d} x} \ell_{p}(x, z) & \text { if } x=y\end{cases}
$$

where as in (3) the variables $x, y$, and $z$ live inside the circle of radius $|p|$ centred at the origin. The function (12) satisfies the obvious symmetry relation $\mathcal{L}_{p}(x, y, z)=\mathcal{L}_{p}(y, x, z)$ and also the functional equation

$$
(x-y) \mathcal{L}_{p}(x, y, z)+(y-z) \mathcal{L}_{p}(y, z, x)+(z-x) \mathcal{L}_{p}(z, x, y)=0,
$$

while the $x=y$ specialization

$$
\mathcal{L}_{p}(x, x, z)=\sum_{n=1}^{\infty} \frac{p^{n} z^{n}}{\left(p^{n}-x\right)^{2}}=\frac{z}{x} \sum_{n=1}^{\infty} \frac{n x^{n}}{p^{n}-z}
$$

contains the $q$-dilogarithmic function [37] as the case $z=1$. In particular, the first two $q$-zeta values [35] are given by

$$
\zeta_{q}(1)=\ell_{p}(1,1) \quad \text { and } \quad \zeta_{q}(2)=\mathcal{L}_{p}(1,1,1) .
$$

Some further arithmetically interesting specializations include

$$
\begin{aligned}
& \mathcal{L}_{p}(1,-1,1)=\sum_{n=1}^{\infty} \frac{p^{n}}{p^{2 n}-1}, \quad \mathcal{L}_{p}(i,-i, 1)=\sum_{n=1}^{\infty} \frac{p^{n}}{p^{2 n}+1}=\frac{\pi_{q}-1}{4}, \\
& \mathcal{L}_{p}\left(e^{2 \pi i / 3}, e^{-2 \pi i / 3}, 1\right)=\sum_{\nu=1}^{\infty} \frac{p^{n}}{p^{2 n}+p^{n}+1}
\end{aligned}
$$


as well as

$$
\mathcal{L}_{p}(-1,-1,1)=\mathcal{L}_{p}(1,1,1)-4 \mathcal{L}_{p^{2}}(1,1,1)=\zeta_{q}(2)-4 \zeta_{q^{2}}(2)
$$

Another generalization of the function (4) is motivated by the fact that it is a "half" of Kronecker's famous identity

$$
\begin{aligned}
\frac{1}{q x} \ell_{p}(x, z / q)-z \ell_{p}(1 /(q x), 1 / z)= & \sum_{\substack{n \in \mathbb{Z}\\
}} \frac{z^{n}}{1-q^{n} x}=\frac{(q, q, x z, q /(x z) ; q)_{\infty}}{(x, q / x, z, q / z ; q)_{\infty}} \\
& |z|<\mid<1
\end{aligned}
$$

and the existence, though much more recent, of an elegant expression for the double Lambert series

$$
\begin{aligned}
\left(\sum_{m, n \geq 0}-\sum_{m, n<0}\right) \frac{q^{m n} y^{m} z^{n}}{1-q^{m+n} x}= & \left(\sum_{\substack{l, m, n \geq 0 \\
|q|<|x|<1, \quad|q|<|y|<1, \quad|q|<|z|<1,}}\right) q^{l m+m n+n l} x^{l} y^{m} z^{n},
\end{aligned}
$$

due to E. Mortenson [26]. This brings to consideration the function

$$
\begin{aligned}
\Lambda_{p}(x, y, z) & =\sum_{l, m, n>0} q^{l m+m n+n l} x^{l} y^{m} z^{n}=x \sum_{m, n>0} \frac{(q y)^{m}(q z)^{n}}{1-q^{m+n} x} \\
& =x \sum_{m, n>0} \frac{y^{m} z^{n}}{p^{m+n}-x}, \quad|y|<|p|, \quad|z|<|p| .
\end{aligned}
$$

We leave a hope that arithmetic studies of the values of the functions may use the techniques of the present note.

\section{Acknowledgements}

I would like to thank Christian Krattenthaler for his earlier communication [21], Eric Mortenson for sharing his remarkable identity from [26] with me as well as Igor Rochev and Armin Straub for useful comments. Further thanks go to the anonymous referee of the journal for suggestions that helped to improve the appearance of the paper.

The work is supported by the Australian Research Council.

Received: 28 January 2016 Accepted: 3 May 2016

Published online: 01 August 2016

\section{References}

1. Amdeberhan, T., Zeilberger, D.: q-Apéry irrationality proofs by q-WZ pairs. Adv. in Appl. Math. 20(2), 275-283 (1998)

2. Amou, M., Katsurada, M., Väänänen, K.: Arithmetical properties of the values of functions satisfying certain functional equations of Poincaré. Acta. Arith. 99(4), 389-407 (2001)

3. Bézivin, J.-P.: Indépendance linéaire des valeurs des solutions transcendantes de certaines équations fonctionnelles. Manuscr. Math. 61, 103-129 (1988)

4. Bézivin, J.-P.: Indépendance linéaire des valeurs des solutions transcendantes de certaines équations fonctionnelles II. Acta. Arith. 55(3), 233-240 (1990)

5. Bézivin, J.-P.: Sur les propriétés arithmétiques d'une fonction entière. Math. Nachr. 190, 31-42 (1998)

6. Bézivin, J.-P.: Fonction de Tschakaloff et fonction q-exponentielle. Acta. Arith. 139(4), 377-393 (2009)

7. Borwein, P.B.: On the irrationality of $\sum\left(1 /\left(q^{n}+r\right)\right)$. J. Number. Theory. 37, 253-259 (1991)

8. Borwein, P.B.: On the irrationality of certain series. Math. Proc. Camb. Phil. Soc. 112, 141-146 (1992)

9. Bundschuh, P., Väänänen, K.: Arithmetical investigations of a certain infinite product. Compos. Math. 91, 175-199 (1994)

10. Bundschuh, P., Väänänen, K.: Linear independence of $q$-analogues of certain classical constants. Results. Math. 47(1-2), 33-44 (2005)

11. Bundschuh, P., Väänänen, K.: Quantitative linear independence of an infinite product and its derivatives. Manuscr. Math. 129(4), 423-436 (2009)

12. Bundschuh, P., Zudilin, W.: Irrationality measures for certain q-mathematical constants. Math. Scand. 101(1), 104-122 (2007)

13. Bundschuh, P., Zudilin, W.: Rational approximations to a $q$-analogue of $\pi$ and some other $q$-series, in "diophantine approximation", Proceedings of the 70th birthday conference in honour of W. M. Schmidt (Vienna, November 2003). In: Schlickewei, H.P., Schmidt, K., Tichy, R.F. (eds.) Developments in mathematics, vol. 16, pp. 123-139. Springer-Verlag, Vienna (2008)

14. Choulet, R.: Des résultats d'irrationalité pour deux fonctions particulières. Collect. Math. 52(1), 1-20 (2001)

15. Clausen, Th.: Beitrag zur Theorie der Reihen. J. Reine. Angew. Math. 3, 92-95 (1828) 
16. Crelle, A.L:: Aufgaben und Lehrsätze, erstere aufzulösen, letztere zu beweisen. J. Reine. Angew. Math. 2, 96-100 (1827)

17. Duverney, D.: À propos de la série $\sum_{n=1}^{+\infty} \frac{x^{n}}{q^{n}-1}$. J. Nombres. Théorie. Bordeaux. 8, 173-181 (1996)

18. Erdős, P.: On arithmetical properties of Lambert series. J. Indian. Math. Soc. 12, 63-66 (1948)

19. Fine, N.J.: Basic hypergeometric series and applications. Math. Surveys. Monogr. vol. 27, Amer. Math. Soc., Providence, RI (1988)

20. Gasper, G., Rahman, M.: Basic hypergeometric series, Encyclopedia of Mathematics and its Applications, vol. 96. Cambridge Univ. Press, Cambridge (2004)

21. Krattenthaler, C.: Personal communication. (17 May 2006)

22. Krattenthaler, C., Rivoal, T., Zudilin, W.: Séries hypergéométriques basiques, $q$-analogues des valeurs de la fonction zêta et formes modulaires. J. Inst. Math. Jussieu. 5(1), 53-79 (2006)

23. Krattenthaler, C., Rochev, I., Väänänen, K., Zudilin, W.: On the non-quadraticity of values of the q-exponential function and related $q$-series. Acta. Arith. 136(3), 243-269 (2009)

24. Matala-aho, T.: On irrationality measures of $\sum_{l=0}^{\infty} d^{\prime} / \prod_{j=1}^{l}\left(1+d^{j} r+d^{2 j} s\right)$. J. Number. Theory. 128(1), 1-16 (2008)

25. Matala-aho, T., Väänänen, K., Zudilin, W.: New irrationality measures for q-logarithms. Math. Comput. 75(254), 879-889 (2006)

26. Mortenson, E.T.: A double-sum Kronecker-type identity, Preprint arXiv:1601.01913 [math.NT] (2016)

27. Pólya, G., Szegő, G.: Problems and theorems in analysis. I. Series, integral calculus, theory of functions. Springer-Verlag, Berlin (1978 \& 1998)

28. Pólya, G., Szegő, G.: Problems and theorems in analysis. II. Theory of functions, zeros, polynomials, determinants, number theory, geometry. Springer-Verlag, Berlin (1976 \& 1998)

29. Pupyrev, Yu. A.: On the linear and algebraic independence of q-zeta values. Math. Notes. 78(3-4), 563-568 (2005)

30. Rochev, I.P.: On the linear independence of the values of some q-series. Izv. Math. 75(1), 177-221 (2011)

31. Rochev, I.P., Väänänen, K.: On a quantitative form of Bézivin's method for q-series. Mosc. J. Comb. Number. Theory. 3(3-4), 169-188 (2013)

32. Smet, C., Van Assche, W.: Irrationality proof of a $q$-extension of $\zeta(2)$ using little $q$-Jacobi polynomials. Acta. Arith. $\mathbf{1 3 8}(2), 165-178$ (2009)

33. Zagier, D.: Vassiliev invariants and a strange identity related to the Dedekind eta-function. Topology 40, 945-960 (2001)

34. Zudilin, W.: On the irrationality measure for a $q$-analogue of $\zeta(2)$. Sb. Math. 193(8), 1151-1172 (2002)

35. Zudilin, W.: Diophantine problems for q-zeta values. Math. Notes. 72(5-6), 936-940 (2002)

36. Zudilin, W.: Heine's basic transform and a permutation group for $q$-harmonic series. Acta. Arith. 111(2), 153-164 (2004)

37. Zudilin, W.: Approximations to $q$-logarithms and $q$-dilogarithms, with applications to q-zeta values. Zap. Nauchn. Sem. S.-Peterburg. Otdel. Mat. Inst. Steklov. POMI 322, Trudy po Teorii Chisel, pp. 107-124 (2005); Reprint in J. Math. Sci. (N. Y.) 137(2), 4673-4683 (2006)

38. Zudilin, W.: A determinantal approach to irrationality. Constr. Approx. (to appear), 10; URL: http://www.link.springer. com/article/10.1007/s00365-016-9333-7

\section{Submit your manuscript to a SpringerOpen ${ }^{\circ}$ journal and benefit from:}

- Convenient online submission

$\checkmark$ Rigorous peer review

- Immediate publication on acceptance

- Open access: articles freely available online

- High visibility within the field

- Retaining the copyright to your article

Submit your next manuscript at springeropen.com 